\title{
Sub-Doppler Measurements and Terahertz Rotational Spectrum of ${ }^{12} \mathrm{C}^{18} \mathrm{O}$
}

\author{
G. Klapper, F. Lewen, R. Gendriesch, S. P. Belov ${ }^{\mathrm{a}}$, and G. Winnewisser
}

I. Physikalisches Institut, Universität zu Köln, Zülpicher Str. 77, D-50937 Cologne, Germany

${ }^{a}$ Institute of Applied Physics, Nizhnii Novgorod 603 600, Russia

Reprint requests to Prof. G. W.; E-mail: winnewisser@ph1.uni-koeln.de

Z. Naturforsch. 56a, 329-332 (2001); received February 23, 2001

\begin{abstract}
The five lowest rotational transitions of ${ }^{12} \mathrm{C}^{18} \mathrm{O}(J=2 \leftarrow 1$ to $J=6 \leftarrow 5)$ have been measured by saturation dip spectroscopy with an experimental accuracy of 1 to $1.5 \mathrm{kHz}$, employing phase-stabilized backward-wave oscillators. The five $J$ rotational transitions cover the frequency range between 219 and $658 \mathrm{GHz}$. In addition, we have measured in the Doppler limited mode the rotational transitions $J=1 \leftarrow 0, J=7 \leftarrow 6$ and $J=8 \leftarrow 7$. The accuracy achieved for the individual frequencies ranges between 5 and $20 \mathrm{kHz}$. Moreover the three rotational transitions $J=16 \leftarrow 15$ to $J=18 \leftarrow 17$ in the frequency region $1.7-2.0 \mathrm{THz}$ were measured with an accuracy of 15 to $30 \mathrm{kHz}$ by using the Cologne sideband spectrometer for terahertz applications COSSTA.
\end{abstract}

Key words: Sub-Doppler Measurement; Rotational Spectrum.

\section{Introduction}

Highly accurate rotational transition frequencies of $\mathrm{CO}$ have been in demand since they serve in the laboratory as an easy but important calibration standard, covering the millimeter wave to the far infrared region. In a continuing effort to provide the most accurate center frequencies for the various isotopomers of $\mathrm{CO}$ throughout the millimeter- and submm wave region, we used saturation dip spectroscopy at frequencies below $1 \mathrm{THz}$ for the following CO isotopomers ${ }^{12} \mathrm{C}^{16} \mathrm{O}[1],{ }^{13} \mathrm{C}^{16} \mathrm{O}$ [2] and ${ }^{13} \mathrm{C}^{18} \mathrm{O}$ [3]. The literature on the determination of laboratory rest frequencies of $\mathrm{CO}$ has always reflected the most recent advances in technological developments concerning measurement accuracy. In contrast to the main isotopomer of carbon monoxides ${ }^{12} \mathrm{C}^{16} \mathrm{O}$, only a few publications of laboratory measurements were reported in the literature for ${ }^{12} \mathrm{C}^{18} \mathrm{O}$. Mainly three groups worked on the topic ${ }^{12} \mathrm{C}^{18} \mathrm{O}$. Winnewisser et al. [4] published spectra of six different $\mathrm{CO}$ isotopomers in the submm-wave region up to $576 \mathrm{GHz}$. This work includes the determination of accurate frequecies of the five lowest rotational transitions of ${ }^{12} \mathrm{C}^{18} \mathrm{O}$ with a statistical measurement uncertainty between 6 and $33 \mathrm{kHz}$. In 1983 Guelachvili et al. [5] published Dunham coefficients of $\mathrm{CO}$ based on fourier Transform measurements of ${ }^{12} \mathrm{C}^{16} \mathrm{O},{ }^{12} \mathrm{C}^{18} \mathrm{O},{ }^{13} \mathrm{C}^{16} \mathrm{O}$ and ${ }^{13} \mathrm{C}^{18} \mathrm{O}$ of vibrational spectra between 1205 and $6335 \mathrm{~cm}^{-1}$. A third line of work concerns Doppler limited measurements from de Natale et al. [6] employing a Fourier Transform spectrometer. The accuracy achieved for these transitions is about 1.2 MHz. For calibration of the spectrometer they used ${ }^{12} \mathrm{C}^{16} \mathrm{O}$ rotational transitions.

\section{Experimental Details}

Two spectrometers were used for the present measurements: the Cologne terahertz spectrometer [7] and the Cologne sideband spectrometer for terahertz appli-

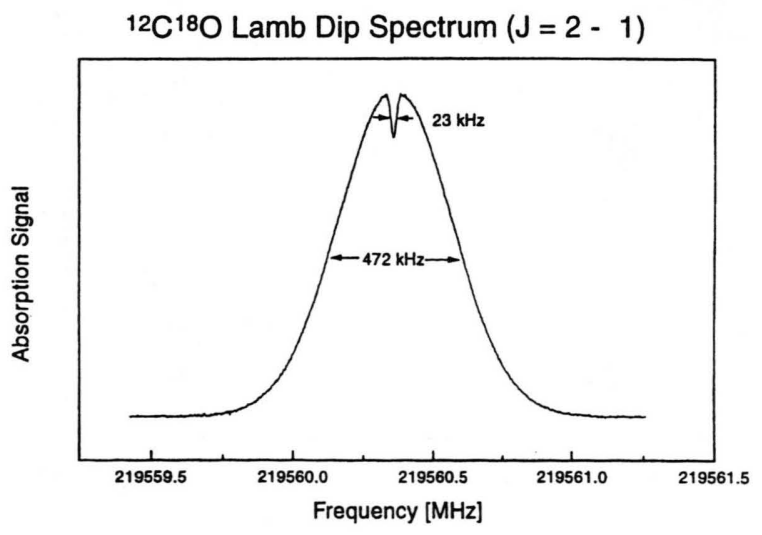

Fig. 1. Lamb dip spectrum of the $J=2 \leftarrow 1$ transition of ${ }^{12} \mathrm{C}^{18} \mathrm{O}$ at $219 \mathrm{GHz}$ on the top of the Doppler-profile. 
${ }^{12} \mathrm{C}^{18} \mathrm{O}$ Lamb Dip Spectrum $(\mathrm{J}=3-2)$

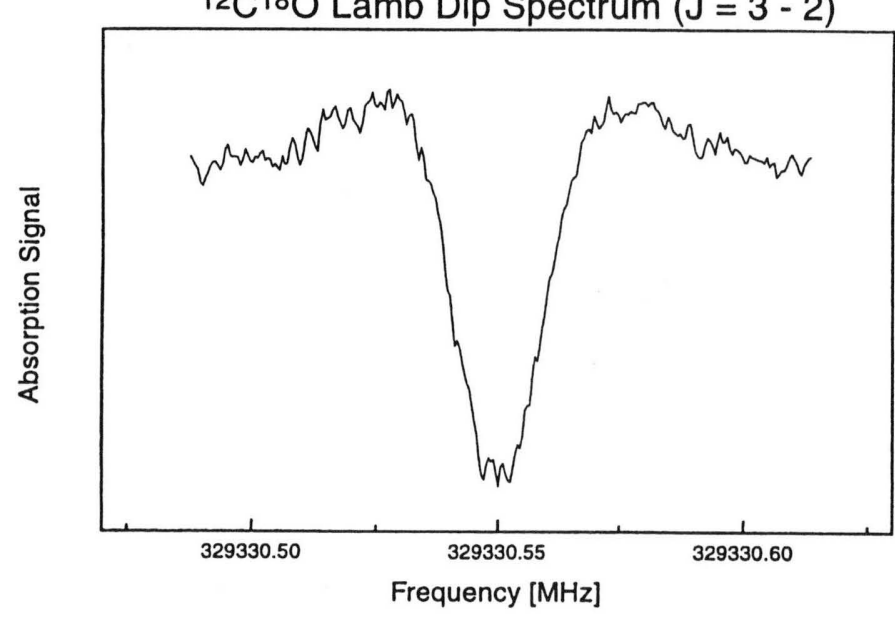

Fig. 2. Lamb dip spectrum of the $J=3 \leftarrow 2$ transition of ${ }^{12} \mathrm{C}^{18} \mathrm{O}$ at $329 \mathrm{GHz}$.

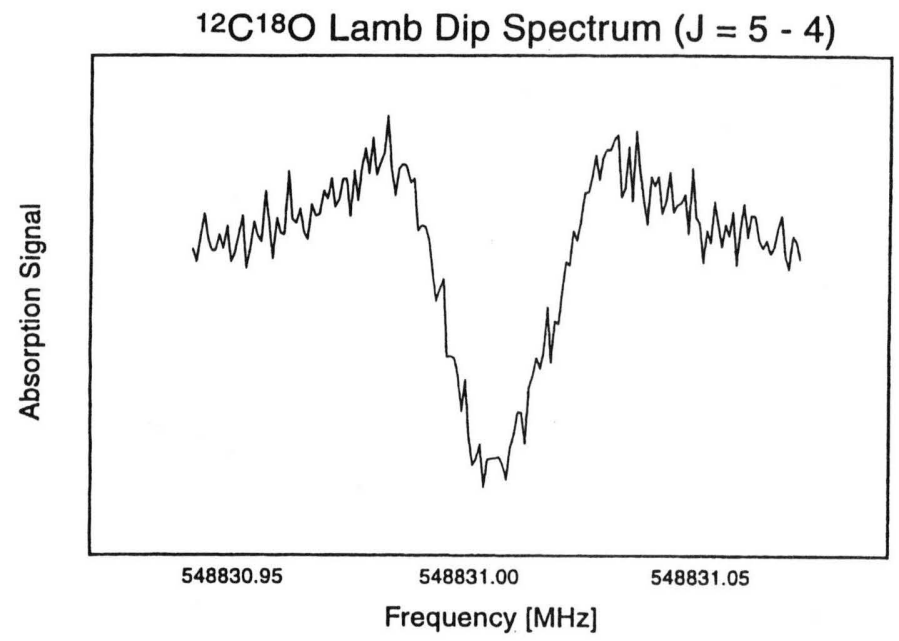

Fig. 4. Lamb dip spectrum of the $J=5 \leftarrow 4$ transition of ${ }^{12} \mathrm{C}^{18} \mathrm{O}$ at $548 \mathrm{GHz}$.
${ }^{12} \mathrm{C}^{18} \mathrm{O}$ Lamb Dip Spectrum $(\mathrm{J}=4-3)$

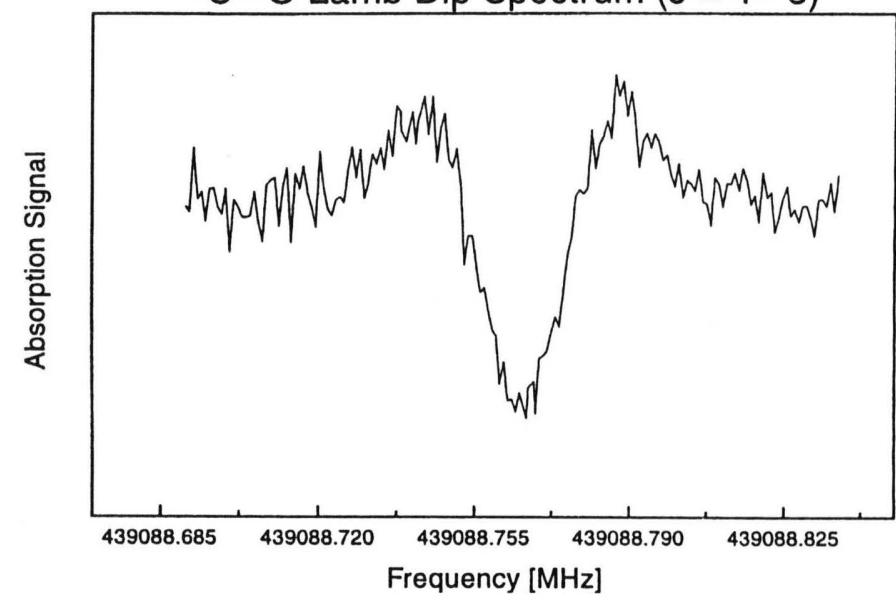

Fig. 3. Lamb dip spectrum of the $J=4 \leftarrow 3$ transition of ${ }^{12} \mathrm{C}^{18} \mathrm{O}$ at $439 \mathrm{GHz}$.

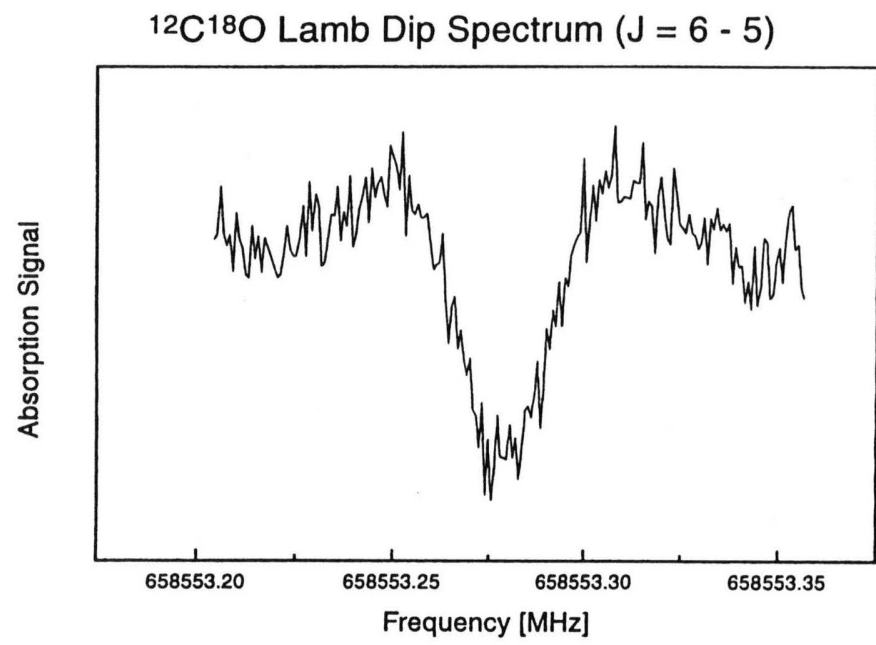

Fig. 5. Lamb dip spectrum of the $J=6 \leftarrow 5$ transition of ${ }^{12} \mathrm{C}^{18} \mathrm{O}$ at $658 \mathrm{GHz}$. 
cations, COSSTA [8]. Both spectrometers operate with phase-locked backward-wave oscillators (BWOs), and in case of the sideband spectrometer with a frequencylocked FIR laser. All reference frequencies are locked against a rubidium frequency standard. The Cologne terahertz spectrometer can be used up to about $1.6 \mathrm{THz}$ [9], whereas the Cologne sideband spectrometer for terahertz applications can presently be tuned to frequencies between 1.7 to $2.0 \mathrm{THz}$. The present measurements on ${ }^{12} \mathrm{C}^{18} \mathrm{O}$ are carried out with both spectrometers. With the Cologne terahertz spectrometer five lines were detected in sub-Doppler resolution $(J=2 \leftarrow 1$ to $J=6 \leftarrow 5$ ) and three lines in Doppler mode $(J=1 \leftarrow 0, J=7 \leftarrow 6$, $J=8 \leftarrow 7)$. Lines measured with the sideband spectrometer were recorded in Doppler limited mode $(J=$ $16 \leftarrow 15, J=17 \leftarrow 16$ and $J=18 \leftarrow 17$ ).

\section{Results}

The newly measured transitions are summarized together with the transitions reported by de Natale et al. [6] in Table 1. The line center frequencies were derived from the measured data points by fitting them to a parabolic function. The achieved measurement accuracies depend on the spectrometer used and on the mode employed. The differences in the accuracies of the Doppler-limited measurements depend on the achieved signal to noise ratio. Doppler lines with good signal to noise ratio can be trusted to $5 \mathrm{kHz}$, whereas the achievable accuracy for unblended, fully resolved Lamb dip measurements in the submillimeter wave region recorded with a good signal to noise ratio estimated to be around $500 \mathrm{~Hz}$ [1]. However, accuracies presented here are estimated to be between 1 to $1.5 \mathrm{kHz}$, depending on the signal to noise ratio.

Figure 1 shows the Lamb dip superimposed on the Doppler line profile. The linewidth of the Doppler profile is about $472 \mathrm{kHz}$, whereas the linewidth of the subDoppler line is about $23 \mathrm{kHz}$. In Figs. 2 to 5 we present a sequence of different rotational transitions recorded with sub-Doppler resolution. Whereas Fig. 6 demonstates the highest frequency Doppler spectrum recorded in this study. The new set of data of ${ }^{12} \mathrm{C}^{18} \mathrm{O}$ was subjected to a least-square fit in which each line was weighted proportionally to the inverse square of its experimental uncertainty. The $\sigma$ of the fit is $2.5 \mathrm{kHz}$. The $0-\mathrm{c}$ values for the frequencies measured by de Natale et al. [6] stay in close agreement with the corresponding $0-c$ values determined in the same reference. In Table 2 we give a
Table 1. Sub-Doppler and Doppler resolved rotational transitions of ${ }^{12} \mathrm{C}^{18} \mathrm{O}$.

\begin{tabular}{llr}
\hline$J^{\prime} \leftarrow J^{\prime \prime}$ & $\begin{array}{l}\text { Obs. Frequencies } \\
{[\mathrm{MHz}]}\end{array}$ & $\begin{array}{c}\mathrm{o} \\
{[\mathrm{kHz}]}\end{array}$ \\
\hline $1 \leftarrow 0$ & $109782.172(20)^{\mathrm{b}}$ & -3.4 \\
$2 \leftarrow 1$ & $219560.3541(15)^{\mathrm{b}}$ & -3.0 \\
$3 \leftarrow 2$ & $329330.5525(15)^{\mathrm{b}}$ & 0.9 \\
$4 \leftarrow 3$ & $439088.7658(10)^{\mathrm{b}}$ & 0.6 \\
$5 \leftarrow 4$ & $548831.0055(10)^{\mathrm{b}}$ & 0.4 \\
$6 \leftarrow 5$ & $658553.2782(10)^{\mathrm{b}}$ & -0.4 \\
$7 \leftarrow 6$ & $768251.5933(50)^{\mathrm{b}}$ & -0.1 \\
$8 \leftarrow 7$ & $877921.9553(60)^{\mathrm{b}}$ & -3.2 \\
$9 \leftarrow 8$ & $987560.2(12)^{\mathrm{c}}$ & -182 \\
$10 \leftarrow 9$ & $1097163.5(12)^{\mathrm{c}}$ & 625 \\
$11 \leftarrow 10$ & $1206724.7(12)^{\mathrm{c}}$ & -748 \\
$12 \leftarrow 1$ & $1316244.4(12)^{\mathrm{c}}$ & 286 \\
$13 \leftarrow 12$ & $1425715.4(12)^{\mathrm{c}}$ & 515 \\
$14 \leftarrow 13$ & $1535133.3(12)^{\mathrm{c}}$ & -476 \\
$15 \leftarrow 14$ & $1644497.3(12)^{\mathrm{c}}$ & 497 \\
$16 \leftarrow 15$ & $1753799.981(30)^{\mathrm{d}}$ & -1.9 \\
$17 \leftarrow 16$ & $1863039.359(20)^{\mathrm{d}}$ & 24.1 \\
$18 \leftarrow 17$ & $1972210.870(15)^{\mathrm{d}}$ & -9.5 \\
$19 \leftarrow 18$ & $2081310.8(12)^{\mathrm{c}}$ & 161 \\
$20 \leftarrow 19$ & $2190332.6(12)^{\mathrm{c}}$ & -2037 \\
$21 \leftarrow 20$ & $2299281.1(12)^{\mathrm{c}}$ & 2198 \\
$22 \leftarrow 21$ & $2408138.2(12)^{\mathrm{c}}$ & -1259 \\
$23 \leftarrow 22$ & $2516913.5(12)^{\mathrm{c}}$ & 1158 \\
$24 \leftarrow 23$ & $2625591.6(12)^{\mathrm{c}}$ & -1981 \\
$25 \leftarrow 24$ & $2734180.4(12)^{\mathrm{c}}$ & 1188 \\
\hline & $\leftarrow 24$ &
\end{tabular}

${ }^{a}$ The errors in parentheses are $1 \sigma$ and given in units of the last quoted digits.

b Frequencies measured with the Cologne terahertz spectrometer. The frequencies of the first five transitions $(J=1 \leftarrow 0)$ measured by Winnewisser et al. [4] in Doppler limited mode agree within quoted errors with the presented frequencies.

c Observed frequencies taken from de Natale et al. [6].

d Frequencies measured with the Cologne sideband spectrometer.

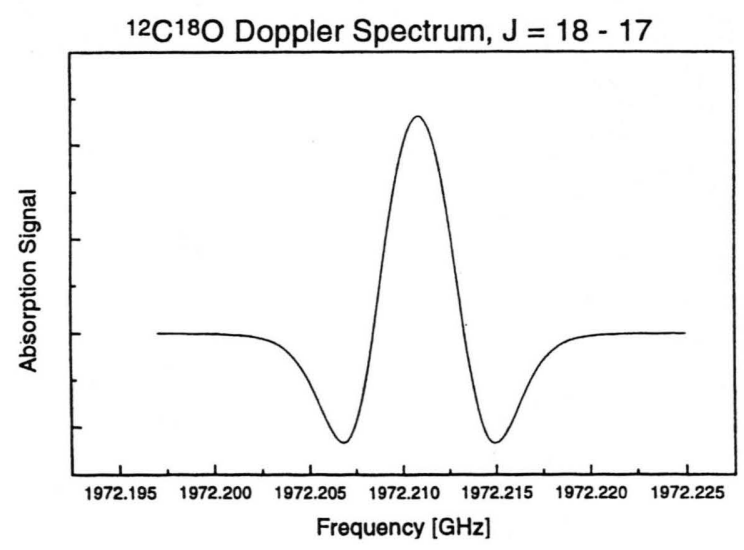

Fig. 6. Doppler spectrum of the $J=18 \leftarrow 17$ transition of ${ }^{12} \mathrm{C}^{18} \mathrm{O}$ at $1972 \mathrm{GHz}$. 
Table 2. Molecular constants of ${ }^{12} \mathrm{C}^{18} \mathrm{O}$.

\begin{tabular}{lccll}
\hline $\begin{array}{l}\text { Con- } \\
\text { stants }\end{array}$ & This work & $\begin{array}{l}\text { M. Winnewisser } \\
\text { et al. [4] }\end{array}$ & $\begin{array}{l}\text { de Natale } \\
\text { et al. [6] }\end{array}$ & Unit \\
\hline$B_{0}$ & $54891.41920(10)$ & $54891.4212(10)$ & $54891.422(5)$ & $\mathrm{MHz}$ \\
$D_{0}$ & $166.4058(27)$ & $166.468(27)$ & $166.308(12)$ & $\mathrm{kHz}$ \\
$H_{0}$ & $0.1458(54)$ & $0.1419^{\mathrm{a}}$ & - & $\mathrm{Hz}$ \\
\hline
\end{tabular}

${ }^{a}$ Values taken from G. Guelachvili et al. [5].

summary of the newly determined constants. The values of the three constants $B_{0}, D_{0}$ and $H_{0}$ generally agree with the corresponding values quoted by Winnewisser et al. [4] and de Natale et al. [6]. On account of the precise sub-Doppler measurements the new constants could be determined more accurately. They reproduce the experimental data very well as can be seen by the $0-c$ values given in Table 1. Because of the higher relative uncer-

[1] G. Winnewisser, S. P. Belov, Th. Klaus, and R. Schieder, J. Mol. Spectrosc. 184, 468 (1997).

[2] G. Klapper, F. Lewen, R. Gendriesch, S. P. Belov, and G. Winnewisser, J. Mol. Spectrosc. 201, 124 (2000).

[3] G. Klapper, F. Lewen, S. P. Belov, and G. Winnewisser, Z. Naturforsch. 55a, 441 (2000).

[4] M. Winnewisser, B. P. Winnewisser, and G. Winnewisser, in Molecular Astrophysics, Series C: Vol. 157, 375-402, by D. Reidel Publishing Company (Edts. G. H. F. Diercksen, W. F. Huebner, and P. W. Langhoff).

[5] G. Guelachvili, G. De Villeneuve, R. Farreng, W. Urban, and J. Verges, J. Mol. Spectrosc. 98, 64 (1983). tainties of the Fourier Transform measurements they were of lower influence to the overall result of the fit.

In summary, precise experimental frequencies of ${ }^{12} \mathrm{C}^{18} \mathrm{O}$ could be determined by sub-Doppler spectroscopy. Accurate frequency predictions can be provided now with the improved molecular constants determined here. Calculated frequencies are available from the authors upon request or under www.ph1.uni-koeln.de.

\section{Acknowledgements}

This work has been supported in part by the Deutsche Forschungsgemeinschaft (DFG) via Grant SFB 301 and 494 and the special funding from the Science Ministry of the Land Nordrhein-Westfalen. The work of S.P.B. at Cologne was made possible by DFG through grants aimed at supporting Eastern and Central European Countries and the republics of the former Soviet Union.

[6] P. De Natale, M. Inguscio, C. R. Orza, and L. R. Zink, Astrophys. J. 370, L53-L55 (1991).

[7] G. Winnewisser, Vibr. Spectrosc. 8, 241 (1995)

[8] R. Gendriesch, F. Lewen, E. Michael, G. Winnewisser, and J. Hahn. J. Mol. Spectrosc. 203, 205 (2000).

[9] F. Maiwald, F. Lewen, V. Ahrens, M. Beaky, R. Gendriesch, A. N. Koroliev, A. A. Negirev, D. G. Paveljev, B. Vowinkel, and G. Winnewisser, J. Mol. Spectrosc. 202, 166 (2000). 